\title{
NETWORK CLASSIFICATION AND IDENTIFICATION BASED ON MACHINE LEARNING
}

\author{
Ganga Gudi \\ Department of Computer Science, \\ KLE's S.Nijalingappa College, \\ Bangalore, India
}

\begin{abstract}
The traffic identification is an important basis for traffic monitoring and data analysis. In this paper, the analysis of network traffic identification is based on machine learning and deep packet inspection. This method uses deep packet inspection technology to identify most network traffic, and improves the accuracy of identification. Machine learning method is used to the identify network traffic with encryption and unknown features, which makes up for the disadvantage of deep packet inspection that cannot identify new applications and encrypted traffic.
\end{abstract}

Keywords-machine learning, DPI, network identification.

\section{INTRODUCTION}

The rapid development of network technology, where users are demanding higher speed and quality of network services. It has become one of the challenges in the field of network operation and maintenance management to manage and control various network traffic.

There are three commonly used methods:

\section{a) Identification Method based on Port Matching}

Identification method classifies the application based on the port number recommended by the IANA (The Internet Assigned numbers Authority). With a large number of network services and development of P2P technology, applications begin to use port and dynamic port technology in order to cross firewalls or avoid other blocking strategies.

\section{b) Identification Method based on Deep Packet Inspection}

The DPI (deep packet inspection) technology detects the load content of IP packet during network interaction or data transmission according to the method of pattern matching, and determines the type of application according.

\section{c) Identification based on Machine Learning}

Due to different application protocols, network data flow has different characteristics in terms of data flow duration,

\author{
Dr Hanumanthappa M \\ Department of Computer Science and \\ Applications, Jnana Bharathi Campus, \\ Bangalore University, India
}

packet length, packet transmission frequency and packet rate. According to these characteristics of network flow, the identification technology in data mining can be used to achieve good traffic identification through machine learning.

\section{NETWORK TRAFFIC IDENTIFICATION METHOD BASED ON MACHINE LEARNING AND DPI TECHNOLOGY}

A. Machine Learning and DPI technical Identification Methods

\section{1) DPI technology}

DPI technology is based on feature field detection. By deeply reading the IP packet load content and reorganizing the application layer information. Then, the data flow content is scanned and detected according to the existing feature library.

\section{a) Machine learning identification methods}

The core of the network traffic identification method based on machine learning is that computer programs can constantly improve their performance with the accumulation of learning experience, so as to complete tasks.
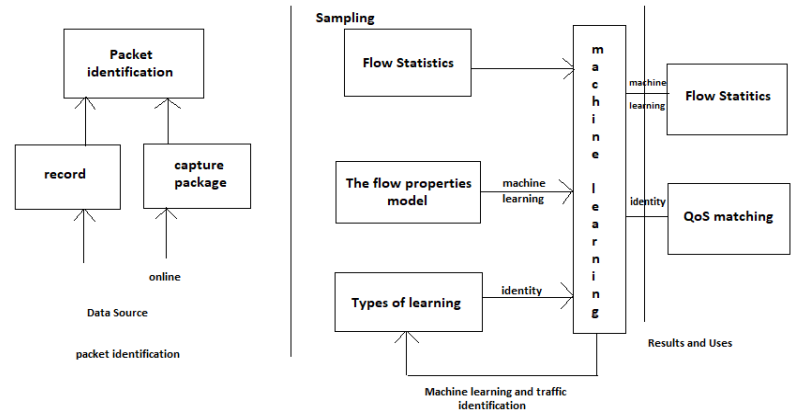

Fig. 1 Flow identification flow chart based on machine learning

\section{B. Algorithm Design}

It is the combination of machine learning and DPI technology. 


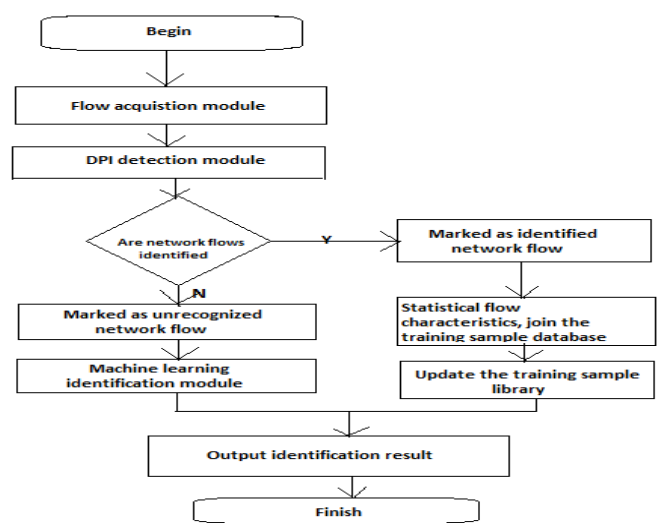

Fig. 2 Network traffic identification process based on DPI and machine learning

- In the DPI detection stage, pattern matching detection is carried out for network data flow according to the protocol feature library loaded.

- Once it is identified by DPI, the flow statistical feature acquisition module sets a fixed collection time and begins to collect the feature information. For the network flow identified by DPI, its flow statistical characteristic information is added to the training sample library.

\section{Algorithm Implementation}

Network traffic identification method is based on DPI and flow statistics features are mainly composed of DPI detection module and machine learning identification module.

\section{a) DPI detection module}

The DPI detection module mainly based on the feature library RuleLib, conducts in-depth analysis of data traffic through pattern matching, and identifies specific application traffic. The detection flow table stores the detected data flow according to the quintuple information.

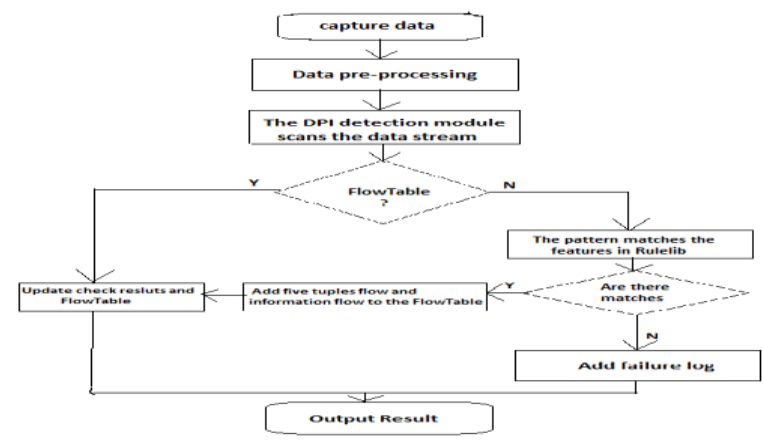

Fig. 3. DPI network traffic identification process.

b) Machine learning identification module

The naive baye's identification method is to determine the category of the sample by calculating the posterior probability. Its basic idea is based on baye's formula and conditional independence assumption in probability theory and the combined probability of attribute and category is used to estimate the category of the new sample.

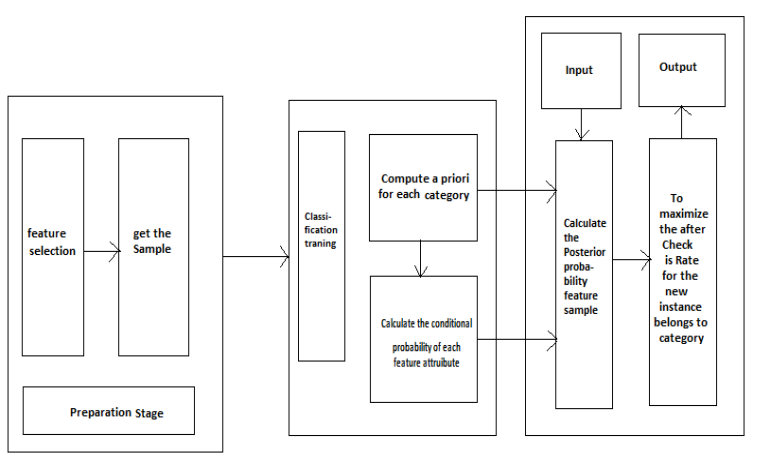

Fig. 4 Naive baye's network traffic identification process

c) Preparation stage.

Feature selection is to get the most effective feature combination on the premise of ensuring the original value of the application data. Feature selection adopts FCBF algorithm to select 8 feature attributes from 248 feature attributes of network flow as feature set.

\section{d) Training stage of classifier}

The naive baye's belongs to the supervised identification method. Matlab is used to analyze the sample data set and calculate the prior probability and conditional probability.

\section{e) Identification stage.}

The classifier is generated according to the decision model of the training phase to realize the online identification of network traffic.

\section{CONCLUSION}

By analyzing two kinds of network traffic identification methods based on feature field and flow statistics, a network traffic identification method based on machine learning and DPI technology is proposed. It uses DPI technology to identify most network traffic, reduces the workload. DPI technology can identify specific application traffic, and improves the accuracy of identification. The machine learning method based on the statistical characteristics of flow is used to assist the identification of network flows with encryption and unknown features, which makes up for the shortcomings of DPI technology in identifying new applications and encrypted traffic, and improves the identification rate of network traffic.

\section{REFERENCES}

1) Xiaoguang Zhang, Lixia Xi and Congpeng Lu Research Optical Network Traffic Based on the 
Content Identification.

2) Research on Network traffic identification Based on Machine Learning Method, 2009.

3) Jingyu Wang, Jiyuan Zhang and, Yuesheng Tan Research of P2P Traffic Identification Based on Traffic Characteristics.

4) Li Wei, Liu Hongyu and, Zhang Xiaoliang - A Network Data Security Analysis Method Based on DPI Technology.

5) RehamTaher El-Maghraby, Nada MostafaAbdElazim and, Ayman M. Bahaa-Eldin - A Survey on Deep Packet Inspection.

6) T. Brinkhoff, "A framework for generating network-based moving objects, "GeoInformatica, vol. 6, no. 2, pp. 153-180, 2002.

7) G.-P. Roh, J.-W. Hwang and B.-K. Yi, "Supporting pattern-matching queries over trajectories on road networks, "IEEE Transactions on Knowledge and Data Engineering, vol, 23, no.11, pp. 1753-1758, 2011.

8) Cheng H, Yan X, Han J, Philip S (2008) Direct discriminative pattern mining. for effective classification.

9) Giannotti F, Nanni M, Pedreschi D - Trajectory pattern mining.

10) M Huang, P. Hu and L. Xia, "A grid based trajectory method for moving objects on fixed network", in Proceedings of the $18^{\text {th }}$ International Conference on GeoInformatics, June 2010.

11) Coppi R, D’Urso P - Fuzzy K-means clustering models for triangular fuzzy time trajectories. Stat Methods Appl 11(1), pp. 22-40.

12) Gudmundsson J, Laube P, Wolle $T$ (2012) Computational movement analysis. Springer handbook of geographic information, pp 725-741. 